\title{
Explicit formulas for a continuous stochastic maturation model. Application to anticancer drug pharmacokinetics/pharmacodynamics.
}

\author{
Djalil Chafaï and Didier Concordet
}

March 2008.

\begin{abstract}
We present a continuous time model of maturation and survival, obtained as the limit of a compartmental evolution model when the number of compartments tends to infinity. We establish in particular an explicit formula for the law of the system output under inhomogeneous killing and when the input follows a time-inhomogeneous Poisson process. This approach allows the discussion of identifiability issues which are of difficult access for finite compartmental models. The article ends up with an example of application for anticancer drug pharmacokinetics/pharmacodynamics.
\end{abstract}

Note: this article is accepted for publication in Stochastic Models (c) Taylor \& Francis, April 2008.

Keywords: Compartmental systems; time lags; stochastic maturation models; inhomogeneous Markov processes; birth and death processes; queueing systems; point processes; Feynman-Kac formulæ, jump processes, delayed equations, pharmacokinetics and pharmacodynamics, modelling of toxicity of anticancer drugs.

AMS-MSC-2000: 92B05；60G07；60K25；60K20；60J80；60J85；47D08；60J25 ; 60J75 .

\section{Introduction}

Compartmental models are widely used in biology and medicine for the modelling of evolution phenomena. In particular, these models are very usual in the pharmacokinetics/pharmacodynamics analysis of toxic effects of anticancer drugs on white blood cells. Several mathematical and computational aspects of such models remain untouched. For instance, at least for catenary chain models, there is no rigorous rule for the choice of the number of compartments, and many identifiability issues are not elucidated. In the present study, we consider the limit of a discrete family of compartmental models when the number of compartments tends to infinity. The obtained limit looks simpler. It provides explicit formulas for the mean occupation of the compartment of interest, and allows the discussion of important identifiability issues. Roughly speaking, we replace a finite catenary chain of compartments with time-inhomogeneous rates by a two compartments time-inhomogeneous model with lag. Much of the article is devoted to the mathematical derivation and analysis of these models. We hope that beyond the mathematical aspects, the main results - illustrated on a simple example at the end of the article - may serve the quantitative biologists.

The mean occupation of the compartment of interest appears as the expectation of an underlying stochastic process. This process is a time-inhomogeneous $M / M / \infty$ queue, with an explicit biological interpretation of its input and output rates. This leads to a nice explicit Binomial-Poisson formula for the instantaneous law. In a way, our approach can be seen as a complement and extension of the boxcartrain models considered for example in [18, 41], see also [20, 27] and references therein. It is also closely linked with "binomial catastrophe models", see for instance [10] and references therein. The novelty is mainly the space-time inhomogeneous killing, the stochastic interpretation in terms of Feynman-Kac's formulæ, and the computation of the output occupation law when the input follows a time-inhomogeneous Poisson process. In practice, the formulas that we derive provide a good compromise between computer time and numerical errors, without loss in interpretation, as suggested 
by the example presented at the end of the article. However, pharmacokinetics/pharmacodynamics populational aspects are outside the scope of this work.

Outline of the rest of the article. The rest of the article is organized as follows.

In Section 1, we briefly introduce the time-inhomogeneous deterministic compartmental systems with linear rates. In particular, we present two stochastic interpretations in terms of particles. The first one is based on interacting time-inhomogeneous $\mathrm{M} / \mathrm{M} / \infty$ queueing systems, whereas the second is based on the occupation of independent random walks on the graph of compartments, subject to birth and death. These interpretations are at the heart of the results of Section 2 regarding limits of catenary chains of compartments.

In section 2, we present a time-inhomogeneous maturation and survival model, and the related counting processes. We show how a finite catenary chain of compartments leads, when the number of compartments tends to infinity, to a simple time-inhomogeneous two compartments model with lag. The main result is given by Theorem 2.4, which provides simple explicit integral formulas for the compartment of interest. These formulas can be seen in turn as a byproduct of Feynman-Kac's formulas, and allow the discussion of identifiability issues in Section 2.3.4.

In Section 3 , the results of Sections 1 and 2 are illustrated on a simple example related to anticancer drug toxicity pharmacokinetics/pharmacodynamics context. Namely, we provide a comparison between classical finite catenary chains of compartments in one hand, and our two compartmental limit with lag in the other hand.

\section{Finite compartmental systems with time-dependent linear rates}

Consider a system of compartments indexed by the finite set $I$. Each compartment contains a quantity of matter. As we will see in the sequel, the amount of matter can be represented by a discrete number, or by a real number, depending on the interpretation chosen. The matter is the subject of transfers between compartments. It can also be created (external inflow) or destroyed (outflow) in each compartment.

The reader may find an accessible introduction to simple compartmental systems with several examples in the recent book [35] by Matis and Kiffe, see also [26] and references therein. The study of the total amount of matter in the system is for instance addressed in [25], see also [34]. The reader may find a study of time-delayed compartmental systems in [37] and [27] and references therein. The literature regarding the stochastic interpretations of time-inhomogeneous compartmental systems is less rich. Let us recall the essential aspects of finite compartmental systems with linear rates.

\subsection{Deterministic systems and linear ordinary differential equations}

We consider here that the amount of matter is represented by a non-negative real number. Let $Q_{i}(t)$ be the amount of matter in compartment $i$ at time $t \geqslant t_{0}$, where $t_{0}$ is the initial time. We denote by $Q(t)$ the vector $i \in I \mapsto Q_{i}(t)$, and we identify the set $\mathbb{R}^{I}$ with $\mathbb{R}^{n}$ where $n:=\operatorname{card}(I)$. Let us consider the dynamics of $(Q(t))_{t \geqslant t_{0}}$ described by the system of linear ordinary differential equations

$$
\forall i \in I, \forall t \geqslant t_{0}, \partial_{t} Q_{i}(t)=\lambda_{i}(t)+\sum_{j \neq i} \rho_{j, i}(t) Q_{j}(t)-Q_{i}(t)\left[\kappa_{i}(t)+\sum_{j \neq i} \rho_{i, j}(t)\right],
$$

with initial condition $Q\left(t_{0}\right)$. Such a dynamics is described by figure 1. Here $\lambda_{i}(t)$ is a creation rate for compartment $i$ (inflow), $\kappa_{i}(t)$ is the destruction rate for compartment $j$ (outflow), and $\rho_{i, j}(t)$ with $i \neq j$ is the transfer rate from compartment $i$ to compartment $j$. These rates are non-negative. Apart from the $\lambda_{i}$ rates, the rates $\kappa_{i}$ and $\rho_{i, j}$ act proportionally to the content of the compartment that gives matter. Note also that a $\kappa_{i}$ with negative values might mimic a proportional auto-inflow. In vector/matrix language, the dynamics of $Q(t)$ is of the form

$$
\partial_{t} Q(t)=\mathbf{M}(t) Q(t)+\lambda(t),
$$


where $\lambda(t)$ denote the "vector" $\left(\lambda_{i}(t) ; i \in I\right)$, and where $\mathbf{M}(t)$ is the matrix defined by

$$
\mathbf{M}_{i, j}(t):= \begin{cases}\rho_{j, i}(t) & \text { if } i \neq j \\ -\sum_{k \neq i} \rho_{i, k}(t)-\kappa_{i}(t) & \text { if } i=j\end{cases}
$$

for any $i, j \in I$. The traditional theory of linear ordinary differential equations gives

$$
Q(t)=\mathbf{R}\left(t, t_{0}\right) Q\left(t_{0}\right)+\int_{t_{0}}^{t} \mathbf{R}(t, u) \lambda(u) d u
$$

for any $t \geqslant t_{0}$, where the resolvent $\mathbf{R}$ is the solution of the matrix linear differential equation $\mathbf{R}\left(t_{0}, t_{0}\right)=$ I and $\partial_{t} \mathbf{R}\left(t, t_{0}\right)=\mathbf{M}(t) \mathbf{R}\left(t, t_{0}\right)$ for any $t \geqslant t_{0}$. In the literature related to compartmental systems, $\mathbf{M}$ is sometimes referred as the "transfer matrix". When $\mathbf{M}$ does not depend on time, $\mathbf{R}(v, u)=$ $\exp ((v-u) \mathbf{M})$. This matrix exponential can be explicitly computed in very special situations where for example card $(I)$ is small or where $\mathbf{M}$ has a nice structure. Usually, the method is to diagonalize $\mathbf{M}$, which leads to an expression of the solution as a linear combination of exponential functions related to the spectrum of $\mathbf{M}$. There is no simple closed formula for $Q(t)$ when the rates depend on time.
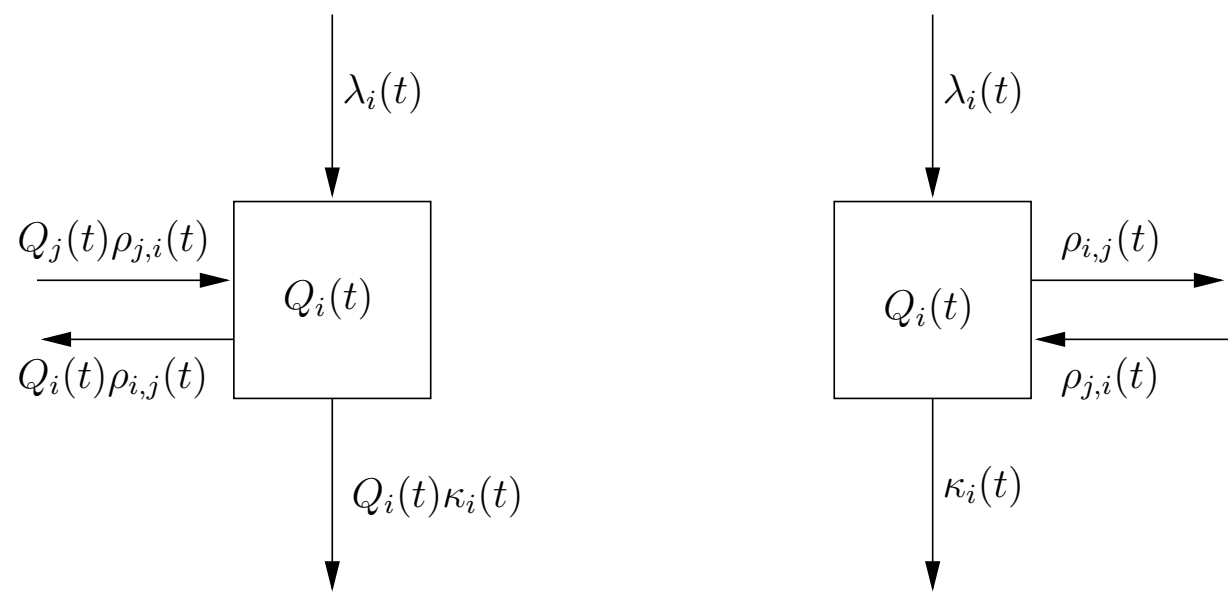

Figure 1: The left hand side diagram shows the flows in a compartmental system. Here, the transfer rates and the outflow depends linearly on the content of the compartment that gives matter. In contrast, the inflow does not depend on the content of the compartments. It is customary to make the $Q_{i}(t)$ and $Q_{j}(t)$ parts of the rates implicit, as show in the right hand side diagram.

Example 1.1 (Finite catenary chain of compartments). It corresponds to a finite system of $n$ compartments, labelled by $I=\{1, \ldots, n\}$, for which $\lambda_{i} \equiv 0$ if $i \neq 1$, and $\rho_{i, j} \equiv 0$ if $j \neq i+1$. It is customary to abridge $\lambda_{1}$ by $\lambda$ and $\rho_{i, i+1}$ by $\rho_{i}$. In such a compartmental system, there is only one external inflow with rate $\lambda$ at the left extremity of the chain. Moreover, the interaction of a compartment is limited to its right neighbor. The topology of this compartmental system is depicted in figure 2. The matrix $\mathbf{M}$ is band diagonal, which makes possible yet tedious the explicit computation of its exponential, when the rates do not depend on time.

\subsection{Stochastic interpretation}

Let us consider a finite system of $n$ compartments, labelled by $I$, and with rates $\lambda, \kappa$, and $\rho$. In the stochastic interpretation, each compartment contains particles, and we denote by $N_{t}^{i}$ the number of particles in compartment $i \in I$ at time $t \geqslant t_{0}$. The particles are indistinguishable, and can be created, destroyed, or can move from one compartment to another, according to a Markovian dynamics of the 


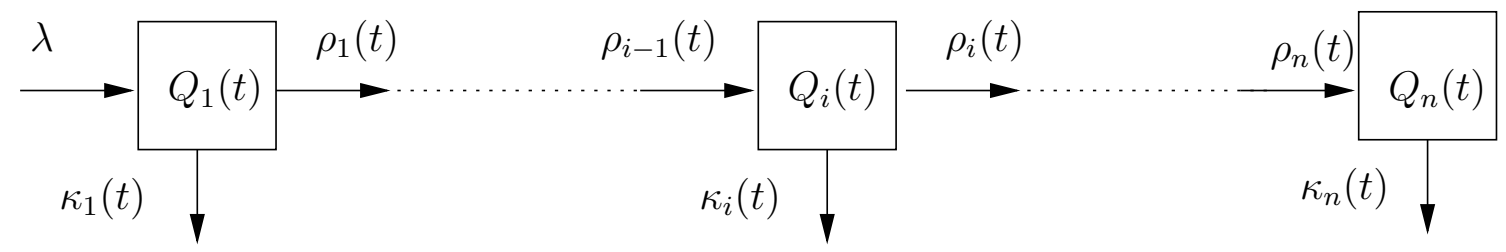

Figure 2: Finite catenary chain of $n$ compartments with single inflow at the chain head.

vector $N_{t}:=\left(N_{t}^{i} ; i \in I\right)$. For a good choice of Markovian dynamics, the average number $\mathbb{E}\left(N_{t} \mid N_{t_{0}}=x\right)$ of particles per compartment is the solution of a system of linear differential equations with initial condition $x$. The reader may find a general presentation of Markov processes and related topics in the book [11] by Ethier \& Kurtz. Kelly gave in [30, sec. 4.5 p. 113-117] an accessible introduction to the stochastic interpretation of compartmental systems. Here we focus on the time-inhomogeneous case.

Theorem 1.2. Let I be a finite set. Let $\left(N_{t}\right)_{t \geqslant t_{0}}$ be a time-inhomogeneous Markov process with state space $E:=\mathbb{N}^{I}$, and generators $\left(\mathbf{L}_{t}\right)_{t \geqslant t_{0}}$. Assume in addition that for any $t \geqslant t_{0}$, the function $x \in E \mapsto$ $A_{t}(x):=\sum_{y \in E} \mathbf{L}_{t, x, y} y$ is affine. For any $x \in E$, and any $t \geqslant s \geqslant t_{0}$, let $Q(s, t, x):=\mathbb{E}\left(N_{t} \mid N_{s}=x\right)$. Then $Q$ is the solution of the linear differential equation

$$
\partial_{t} Q(s, t, x)=A_{t}(Q(s, t, x)),
$$

for any $t \geqslant s \geqslant t_{0}$, with initial condition $Q(s, s, x)=x$.

Proof. The result is a consequence of Chapman-Kolmogorov equations. Namely, for any $t \geqslant s \geqslant t_{0}$ and any function $f: E \rightarrow \mathbb{R}$, let $P_{s, t}(f)$ be the function $E \rightarrow \mathbb{R}$ defined by $P_{s, t}(f)(x):=\mathbb{E}\left(f\left(N_{t}\right) \mid N_{s}=x\right)$. In particular, $P_{s, s}(f)=f$. The Markovianity of the process is captured by the Chapman-Kolmogorov equation which writes $P_{s, u}\left(P_{u, t}(f)\right)=P_{s, t}(f)$ for any $t \geqslant u \geqslant s \geqslant t_{0}$. Recall that $\mathbf{L}_{t}$ acts linearly on $f$ as a matrix. We denote by $\mathbf{L}_{t} f$ the function $E \rightarrow \mathbb{R}$ defined by $\left(\mathbf{L}_{t} f\right)(x):=\sum_{y \in E} \mathbf{L}_{t, x, y} f(y)$. The definition of $\mathbf{L}$ gives

$$
\left(\mathbf{L}_{t} f\right)(x):=\lim _{\varepsilon \rightarrow 0^{+}} \frac{P_{t, t+\varepsilon}(f)(x)-P_{t, t}(f)(x)}{\varepsilon} .
$$

This yields, by using the Chapman-Kolmogorov equation, to the "forward equation"

$$
\partial_{t} P_{s, t}(f)(x):=\lim _{\varepsilon \rightarrow 0^{+}} \frac{P_{s, t+\varepsilon}(f)(x)-P_{s, t}(f)(x)}{\varepsilon}=P_{s, t}\left(\mathbf{L}_{t}(f)\right)(x) .
$$

The desired results follows immediately by considering the ad-hoc $f$ function. Namely, for any $i \in I$, consider the function defined by $f(x)=x_{i}$ for any $x \in E$. One has $\left(\mathbf{L}_{t} f\right)(x)=A_{t}(x)_{i}$. Since $A_{t}$ in affine and $P_{s, t}$ is Markov, they commute and we get $P_{t}\left(\mathbf{L}_{t} f\right)(x)=A_{t}\left(P_{s, t}(f)(x)\right)_{i}$.

Note that the Chapman-Kolmogorov equation which appears in the proof of theorem 1.2 gives also the "backward" equation $\partial_{s} P_{s, t}(f)(x)=-\mathbf{L}_{s}\left(P_{s, t}(f)\right)(x)$, which yields the system of linear differential equations

$$
\partial_{s} Q(s, t, x)=-\sum_{y \in E} \mathbf{L}_{s, x, y} Q(s, t, y)
$$

with initial condition $Q(s, s, x)=x$. Of course, when the process is time-homogeneous, $\mathbf{L}_{t}$ does not depend on time anymore and $P_{s, t}=P_{t_{0}, t_{0}+t-s}$. Thus, in that case, $P_{u}(\mathbf{L}(f))=\mathbf{L}\left(P_{u}(f)\right)$ for any $u \geqslant t_{0}$, which makes the forward and backward equations identical up to a sign.

Let us give now an $\mathbf{L}_{t}$ matrix on $E$ such that $A_{t}$ is exactly the affine function of the deterministic compartmental system (2) constructed from the triplet of rates $\lambda(t), \kappa(t)$ and $\rho(t)$. We identify the 
countable space $E:=\mathbb{N}^{I}$ with $\mathbb{N}^{n}$ where $n:=\operatorname{card}(I)$. We denote by $e_{1}, \ldots, e_{n}$ the canonical basis of $\mathbb{R}^{n}$, embedded in $E$. For any $x, y \in E$ with $x \neq y$, we set

$$
\mathbf{L}_{t, x, y}:=\left\{\begin{array}{ll}
\lambda_{i}(t) & \text { if } y=x+e_{i} \text { for } i \in I \text { (birth of a particle) } \\
x_{i} \rho_{i, j}(t) & \text { if } y=x-e_{i}+e_{j} \text { for } i \neq j \text { in } I \text { (transfer of a particle) } \\
x_{i} \kappa_{i}(t) & \text { if } y=x-e_{i} \text { for } i \in I \text { (death of a particle) } \\
0 & \text { otherwise }
\end{array} .\right.
$$

The diagonal terms are such that the sum over each row is zero. The transition related to $\rho$ can equivalently expressed as $x_{j} \rho_{j, i}(t)$ if $y=x-e_{j}+e_{i}$ for $j \neq i$. Recall that $N_{t}$ is an $n$-components vector which represents the amount of particles in each of the $n$ compartments at time $t$. A simple computation shows that for any $x \in E$ and any $i \in I$,

$$
A_{t}(x)_{i}:=\sum_{y \in E} \mathbf{L}_{t, x, y} y_{i}=\lambda_{i}(t)+\sum_{j \in I, j \neq i} x_{j} \rho_{j, i}(t)-x_{i}\left[\kappa_{i}(t)+\sum_{j \in I, j \neq i} \rho_{i, j}(t)\right] .
$$

We recognize immediately the equation (2) of the deterministic compartmental system.

\subsubsection{Interpretation as interacting $\mathrm{M} / \mathrm{M} / \infty$ queues}

The generator (3) suggests an interpretation of the process $\left(N_{t}\right)_{t \geqslant t_{0}}$ as an $n$-dimensional $\mathrm{M} / \mathrm{M} / \infty$ queueing system with interactions. In such a picture, each particle is a client, and each compartment is an $\mathrm{M} / \mathrm{M} / \infty$ queue. The quantity $N_{t}^{i}$ is thus the number of customers in the $i^{\text {th }}$ queue at time $t$. For each compartment $i \in I$, and in absence of interaction $(\rho \equiv 0)$, the arrival rate is $\lambda_{i}$, and the service rate is $\kappa_{i}$. Since the queue is $\mathrm{M} / \mathrm{M} / \infty$, each new client gets immediately its own dedicated server, which explains the coefficient $x_{i} \kappa_{i}$ in $\mathbf{L}$. The interaction $\rho$ allows the clients to move from queue $i$ to queue $j$ with rate $x_{i} \rho_{i, j}$. Thus, in presence of interactions, the arrival rate in queue $i$ is $\lambda_{i}+\sum_{j} x_{j} \rho_{j, i}$ and the service rate is $x_{i} \kappa_{i}+x_{i} \sum_{j} \rho_{i, j}$. The clients in the queues are not ordered since these queues are of $\mathrm{M} / \mathrm{M} / \infty$ type. The random vector $N_{t}$ gives the number of clients in each queue at time $t$. The dynamics of $\left(N_{t}\right)_{t \geqslant t_{0}}$ can be thus interpreted as $n$ interacting $\mathrm{M} / \mathrm{M} / \infty$ queues, e.g. as $n$ interacting birth and death processes on $\mathbb{N}$. These interacting queues can be seen as special Markovian interacting particle systems, see [9, 33]. In statistical mechanics, they constitute a "zero-range dynamics on the graph of compartments", see [3]. For the reader familiar with queueing systems, these interacting queues can be seen as time-inhomogeneous "Jackson networks" [23, 24, 40]. These queues are "in tandem" in the case of a catenary chain of compartments with killing only at the end of the chain.

In particular, with such an interpretation in mind, a one compartment system is equivalent to a single $\mathrm{M} / \mathrm{M} / \infty$ queue. More generally, a catenary chain of $n$ compartments is equivalent to a system of $n$ interacting $\mathrm{M} / \mathrm{M} / \infty$ queues, in which only the first one has an external inflow, the others being inflowed by the clients that leave the preceding queue due to the interaction.

\subsubsection{Interpretation as independent particles on the graph of compartments}

Following [30, sec. 4.5 p. 113-117], by removing the indistingishability of the particles assumed in the preceding interpretation, $N_{t}$ is the occupation vector of the compartments at time $t$ for a superposition of independent particles moving in $I$. These particles are subject to birth ( $\lambda$ rate) and death ( $\kappa$ rate), in addition to their motion in the system of compartments viewed as an oriented graph with $n$ vertices. This graph is a subset of $I \times I$, and $\rho_{i, j} \equiv 0$ means that the edge $(i, j)$ does not exist in the graph. In other words, $\rho$ defines an oriented graph, i.e. a topology on the system of compartments.

Note that when $n=1$ (trivial graph), we just have constructed the $\mathrm{M} / \mathrm{M} / \infty$ queue from independent particles with survival rate $\kappa$, arriving according to an independent Poisson point process of intensity $\lambda$. When $n \geqslant 1$ and $\rho \equiv 0$, the process consists in $n$ independent $\mathrm{M} / \mathrm{M} / \infty$ queues. 


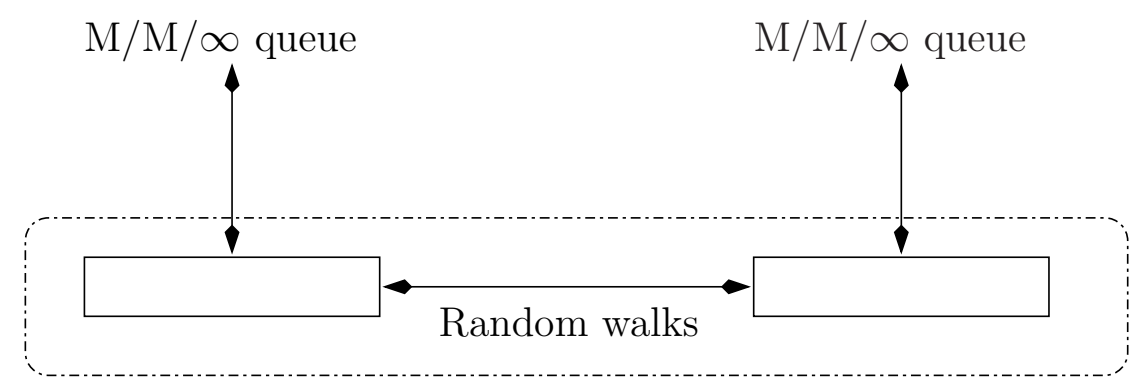

Graph of compartments

Figure 3: The two stochastic interpretations of finite compartmental systems with linear rates. Interacting $\mathrm{M} / \mathrm{M} / \infty$ queues versus independent random walks on the graph of compartments subject to birth and death.

In this interpretation, the creation of particles on the $n$ sites is obtained with $n$ independent Poisson point processes, one for each external inflow. The independence of the particles moving on the graph is a consequence of the linearity in $x$ of the coefficients related to the $\rho$ rates in $\mathbf{L}_{x, y}$. The single particle dynamics involves the $\rho$ rates for its motions. The $\kappa$ rates are incorporated by the addition of an external cemetery state to the system, whereas the $\lambda$ rates are incorporated by using an independent Poisson point process and independent copies of the particle. It is known that adding an external cemetery state leads to Feynman-Kac type formulas related to a very simple potential, see [8] for instance.

We have thus two stochastic interpretations of the dynamics (3) of $\left(N_{t}\right)$, as presented by figure 3 . The first one is "vertical" and corresponds to interacting birth and death processes in $\mathbb{N}(\mathrm{M} / \mathrm{M} / \infty$ queues subject to interactions), whereas the second one is "horizontal" and corresponds to independent particles moving on the graph of compartments (random walks on $I$ subject to birth and death). Both hold for time-dependent rates.

\section{A continuous stochastic maturation model}

In the sequel, we consider a time-inhomogeneous stochastic maturation model with killing, which provides a Poisson-Binomial formula for the occupation law of the maturation system. The probability of survival in such a model can be for instance the probability of traversal of a finite catenary chain of compartments. We show that this probability has a nice explicit formula when the length of the chain tends to infinity. Such a result is in accordance with the stochastic interpretation of finite compartmental systems presented in Section 1, in terms of time-inhomogeneous $M / M / \infty$ queues.

\subsection{Counting mature particles under killing and Poisson production}

Consider a maturation system in which the maturation takes a deterministic duration $\tau>0$. A particle which begins its maturation at time $T \in \mathbb{R}$ achieves its maturation at time $T+\tau$. We say that $T+\tau$ is the maturation time of the particle. Assume in addition that the particle can die during the maturation process, with probability $1-p(T)$, where $p(T) \in[0,1]$ is a deterministic real number which may depend on $T$. In such a maturation with killing scheme, a particle which begins its maturation at time $T \in \mathbb{R}$ can either die with probability $1-p(T)$, or survive with probability $p(T)$ and achieve its maturation at time $T+\tau$. Now, consider independent particles which begin their maturation at times $T_{0}, T_{1}, \ldots$ These particles survive with probabilities $p\left(T_{0}\right), p\left(T_{1}\right), \ldots$, and achieve their maturation at times $M_{0}=T_{0}+\tau, M_{1}=T_{1}+\tau, \ldots$ The following Lemma is a consequence of the stability by thinning of Poisson point processes, see for instance [31] or [19]. 
Lemma 2.1. Suppose that $T_{0}, T_{1}, \ldots$ are random and distributed according to a Poisson process with intensity $\lambda: \mathbb{R} \rightarrow \mathbb{R}_{+}$. Assume that this random process is independent from the maturation process of the particles. The maturation times $M_{0}, M_{1}, \ldots$ follow a Poisson point process on $\mathbb{R}$ with intensity $\lambda^{*}: \mathbb{R} \rightarrow \mathbb{R}_{+}$given by

$$
\lambda^{*}(t):=\lambda(t-\tau) p(t-\tau) .
$$

In other words, the number of mature particles produced during a bounded time interval $I \subset \mathbb{R}$ follows a Poisson distribution with mean

$$
\int_{I} \lambda^{*}(u) d u=\int_{I} \lambda(u-\tau) p(u-\tau) d u
$$

Moreover, for any disjoint and bounded time intervals $I$ and $J$, the number $N_{I}$ and $N_{J}$ of mature particles produced during these time intervals are independent Poisson random variables. The reader familiar with point processes may notice that the resulting point process is thus an inhomogeneous marked Poisson point process, with Bernoulli marks.

Let us assume now in addition that independently, each mature particle survives after maturation during a random duration with intensity $\mu: \mathbb{R}_{+} \rightarrow \mathbb{R}_{+}$. In other words, given that a mature particle is still alive at time $s$, and if $S$ is its remaining survival duration, then $\mathbb{P}(S>t)=\exp \left(-\int_{s}^{t} \mu(w) d w\right)$ for any $t \geqslant s$. The following Theorem expresses the law of the number $N_{t}$ of mature particles still alive at time $t \in \mathbb{R}_{+}$. Figure $\mathbb{6}$ gives a schematic view of the system.

The following Theorem is a direct consequence of Lemma 2.1, but can also be seen alternatively as a special case of a result of Keilson \& Servi [29] on time-inhomogeneous M/G/ $\infty$ queuing processes (see also [13, 21, 43] for related problems). Here the resulting counting process $\left(N_{t}\right)_{t \geqslant 0}$ is a timeinhomogeneous $\mathrm{M} / \mathrm{M} / \infty$ queue. The reader may find a general introduction to Queuing Processes in many books such as [1, 国, 12, 30, 31, 40].

Theorem 2.2. For any times $0 \leqslant s \leqslant t$ and any $m \in \mathbb{N}$,

$$
\mathcal{L}\left(N_{t} \mid N_{s}=m\right)=\mathcal{B}(m, \alpha(s, t)) * \mathcal{P}(\beta(s, t))
$$

where

$$
\alpha(s, t):=\exp \left(-\int_{s}^{t} \mu(w) d w\right) \quad \text { and } \quad \beta(s, t):=\int_{s}^{t} \lambda(u-\tau) p(u-\tau) \alpha(u, t) d u .
$$

In particular, $\mathbf{E}\left(N_{t} \mid N_{s}=m\right)=m \alpha(s, t)+\beta(s, t)$ for any $0 \leqslant s \leqslant t$ and any $m \in \mathbb{N}$.

Note that there are three sources of randomness here, which are supposed independent: the initial time of maturation, the survival during the maturation, and the survival duration after maturation.

\subsection{Modelling the maturation process itself for one particle}

Let us consider a single particle which begins its maturation at time $T \in \mathbb{R}$. This section is devoted to the modelling of the maturation and survival process itself, by means of a Markovian dynamics on the time interval $[T,+\infty)$. In particular, it provides an explicit formula of the probability of survival during maturation $p(T)$.

\subsubsection{Finite state model}

We begin by considering a maturation model which consists in $n+1$ maturation steps, with possible killing. More precisely, define the finite set

$$
\mathcal{S}^{(n)}:=\{0,1, \ldots, n\} \cup\{c\}
$$

where $c \notin\{0,1, \ldots, n\}$ will serve as a cemetery state. The maturation of the particle is modelled by a motion from state 0 to state $n$. State $n$ corresponds to "maturity". The killing rate is modelled by a function

$$
\kappa^{(n)}: \mathbb{R} \times\{0, \ldots, n\} \rightarrow \mathbb{R}_{+}
$$




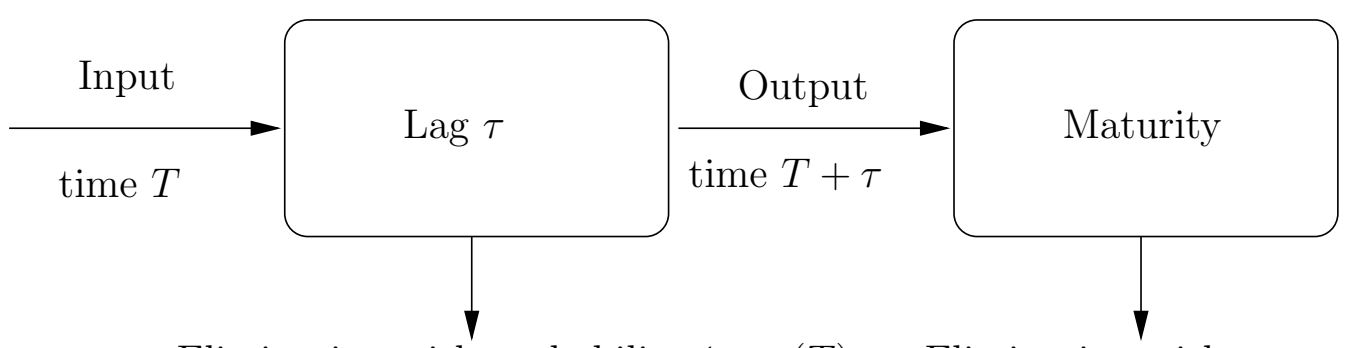

Elimination with probability $1-p(T) \quad$ Elimination with rate $\mu$

Figure 4: Schematic view of a maturation system with killing. Recall that $\tau$ is the maturation duration. The quantity $p(T)$ is the survival probability of a particle which has begun its maturation at time $T$. The quantity $\mu$ is the survival rate of a mature particle. The left hand side compartment (LC) corresponds to a Poisson deformation box with lag, whereas the right hand side compartment (RC) corresponds to an $\mathrm{M} / \mathrm{M} / \infty$-like counting process. The output of the $\mathrm{LC}$ is a point process which serves as an input for the RC.

which is smooth in the first variable (time). This killing rate may thus vary in time and in space. The maturation rate is modelled by a constant positive real number $\rho^{(n)}$. Consider now the continuous time Markov process $\left(X_{t}^{(n)}\right)_{t>T}$ with state space $\mathcal{S}^{(n)}$, and generator given for any $i \neq j$ in $\mathcal{S}^{(n)}$ and any $t \geqslant T$ by

$$
\left(\mathbf{L}_{t}^{(n)}\right)_{i, j}=\left\{\begin{array}{ll}
\rho^{(n)} & \text { if } i \neq c \text { and } j=i+1 \\
\kappa^{(n)}(t, i) & \text { if } i \neq c \text { and } j=c \\
0 & \text { otherwise }
\end{array} .\right.
$$

The diagonal terms of the matrix $\mathbf{L}^{(n)}$ are such that the sum over each row is 0 . The dynamics of the maturation process $\left(X_{t}^{(n)}\right)_{t \geqslant T}$ can be read directly on the expression of the generator above. Namely, at time $t$ and from state $i$, the process can only move to state $i+1$ with rate $\rho^{(n)}$, or die (being killed) with rate $\kappa(t, i)$ and placed in the cemetery state $c$ from which it cannot escape. The maturation steps are depicted by figure 5. In absence of killing, i.e. when $\kappa^{(n)} \equiv 0$, the process $\left(X_{t}^{(n)}\right)_{t \geqslant T}$ is a simple Poisson process of intensity $\rho^{(n)}$, stopped when it reaches state $n$.

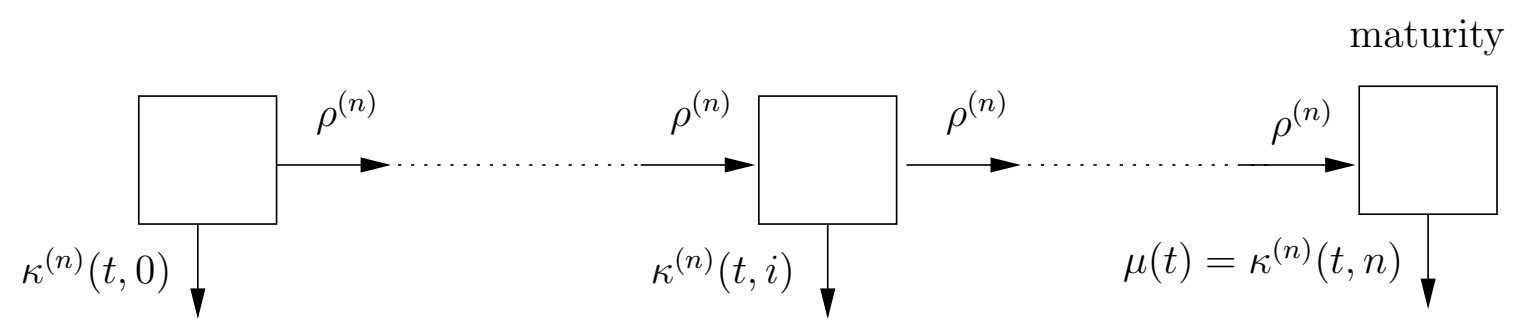

Figure 5: A catenary chain of compartments with one-way flow and possible killing, used as a maturation model maturation (or evolution) rate $\rho^{(n)}$, and killing rate $\kappa^{(n)}$. The down arrows lead to the cemetery state, which is not represented here.

In such a maturation with killing model, we are interested in the mean occupation of the state $n$ for a Poissonian number of such processes evolving independently. One can define $p(T)$ as the probability of hitting state $n$ starting from state 0 :

$$
p(T)=\mathbb{P}\left(\exists t>T ; X_{t}^{(n)}=n \mid X_{T}^{(n)}=0\right) \quad \text { and } \quad \mu(t)=\kappa^{(n)}(t, n) .
$$

However, the corresponding maturation time is random, depends on $T$, and is given by

$$
\tau=\inf \left\{t>T ; X_{t}^{(n)}=n\right\}
$$


on the event $\left\{X_{T}^{(n)}=0, \exists t>T ; X_{t}^{(n)}=n\right\}$. We will see in the sequel that letting $n$ tends to $\infty$ provides a very simple explicit formula for $p(T)$ and makes $\tau$ deterministic, without any loss in interpretation.

\subsubsection{Continuous state limit model}

It is possible to express the law of $\left(X^{(n)}\right)_{t \geqslant T}$ by mean of a Feynman-Kac formula. Rather than using this approach, we prefer to consider now the limit process obtained by letting the number of compartments $n$ tends to $\infty$. We will use a scaling which corresponds to the convergence

$$
\left\{\frac{0}{n}, \frac{1}{n}, \ldots, \frac{n}{n}\right\} \rightarrow[0,1]
$$

Namely, assume that $\rho^{(n)}=n \rho$ for some positive constant $\rho$. Assume that

$$
\lim _{n \rightarrow \infty} \sup _{i \in\{0, \ldots, n\} ; t \in \mathbb{R}}\left|\kappa^{(n)}(t, i)-\kappa(t, i / n)\right|=0
$$

for some bounded piecewise continuous function

$$
\kappa: \mathbb{R} \times[0,1] \rightarrow \mathbb{R}_{+} .
$$

Theorem 2.3. Let $x \in[0,1]$. On the event $\left\{X_{T}^{(n)}=\lfloor n x\rfloor\right\}$, and with the convention $c / n=c$, the rescaled process $\left(n^{-1} X_{t}^{(n)}\right)_{t \geqslant T}$ converges in law, when $n$ goes to $\infty$, toward the Markov process $\left(X_{t}\right)_{t \geqslant T}$ with state space $\mathcal{S}:=[0,1] \cup\{c\}$ defined for any $T \leqslant s \leqslant t$ by

$$
\mathcal{L}\left(X_{t} \mid X_{s}=x\right)= \begin{cases}p(s, t, x) \delta_{x(t-s)}+(1-p(s, t, x)) \delta_{c} & \text { if } x \neq c \\ \delta_{c} & \text { if } x=c\end{cases}
$$

where $x(u):=\min (1, x+\rho(u-T)), p(u, v, x):=\exp \left(-\int_{u}^{v} \kappa(w, x(w-u)) d w\right)$, and $p(u, v, c):=0$ for any $x \neq c$.

Proof. Consider the natural inclusion $\pi_{n}: \mathcal{S}^{(n)} \rightarrow \mathcal{S}$ defined by $\pi_{n}(i)=i / n$ for any $i \in\{0, \ldots, n\}$ and $\pi_{n}(c)=c$. By this way, any function $f: \mathcal{S} \rightarrow \mathbb{R}$ induces naturally a function $f\left(\pi_{n}\right)$ from $\mathcal{S}^{(n)}$ to $\mathbb{R}$, and one has

$$
\mathbf{L}_{t}^{(n)}\left(f\left(\pi_{n}\right)\right)(i)=n \rho\left(f\left(\frac{i+1}{n}\right)-f\left(\frac{i}{n}\right)\right)+\kappa\left(t, \frac{i}{n}\right)\left(f(c)-f\left(\frac{i}{n}\right)\right) .
$$

By following step by step the classical argument based on Taylor's formulas presented in [11, p. 1-5], one can show that the Markov process $\left(n^{-1} X_{t}^{(n)}\right)_{t \geqslant T}$ converges in law, when $n$ goes to $\infty$, toward the Markov process $\left(X_{t}\right)_{t \geqslant T}$ with state space $\mathcal{S}:=[0,1] \cup\{c\}$ and infinitesimal generators

$$
\mathbf{L}_{t}(f)(x)= \begin{cases}\rho f^{\prime}(x)+\kappa(t, x)(f(c)-f(x)) & \text { if } x \neq c \\ 0 & \text { if } x=c\end{cases}
$$

defined for continuous functions $f: \mathcal{S} \rightarrow \mathbb{R}$ which are smooth on $[0,1]$ and vanish at the boundaries. The scaling method that we used is related to the fluid limits of Queuing Theory (see for instance in [40, ch. 9]) and the hydrodynamic limits of interacting particles systems in Statistical Mechanics (see for instance [32]). The obtained generator $\mathbf{L}_{t}$ above is the addition of a deterministic constant drift term $\rho f^{\prime}(x)$ together with a random space-time inhomogeneous killing term $\kappa(t, x)(f(c)-f(x))$. A Feynman-Kac formula or a direct check gives the desired expression of the law. Actually, the addition of a cemetery state corresponding to a killing term to a Markov process always gives rise to a Feynman-Kac formulae, see [8, 28]. In some ways, our case (6) is the simplest one: killing term + deterministic generator (constant drift). 
The interpretation in terms of the position $X_{t}$ of the particle is quite clear: the particle is moving from left to right on the interval of positions $[0,1]$ at constant speed $\rho$. The particle stops its motion when (and if) it reaches the right extremity of $[0,1]$. At any time $t \in \mathbb{R}_{+}$and any position $x \in[0,1]$, it can be killed (and thus placed in the cemetery state $c$ ) with rate $\kappa(t, x)$. A particle is mature when it reaches the right extremity 1 of $[0,1]$. The positions $[0,1)$ correspond to the steps of maturation. For any $t \in \mathbb{R}$ and any $x \in[0,1]$, let us define $g(t, x):=\kappa(t, x) \mathrm{I}_{[0,1)}(x)$ and $\mu(t):=\kappa(t, 1)$ in such a way that

$$
\kappa(t, x)=g(t, x) \mathrm{I}_{[0,1)}(x)+\mu(t) \mathrm{I}_{\{1\}}(x) .
$$

Function $g$ gathers the killing rate during maturation, whereas function $\mu$ captures the killing rate after maturation. In the settings of Section 2.1, the process $\left(X_{t}\right)_{t \geqslant T}$ corresponds to a maturation model for which

$$
\mu(t)=\kappa(t, 1) \quad \text { and } \quad p(T)=p(T, T+\tau, 0)=\exp \left(-\int_{0}^{\tau} g(T+w, \rho w) d w\right)
$$

where the "maturation time" $\tau$ is deterministic, independent of $t$, and given by

$$
\tau:=\frac{1}{\rho} .
$$

When the particle is not killed, i.e. on the event $\left\{X_{T}=0, \exists s>0 ; X_{s}=1\right\}, \tau$ is exactly the deterministic duration taken by the particle to reach state 1 from state 0 (at constant speed $\rho$ ).

\subsection{The resulting counting process}

Consider the maturation system with killing modelled by the $\left(X_{t}\right)$ process introduced above, with maturation speed $\rho$ and killing rate $\kappa: \mathbb{R} \times[0,1] \rightarrow \mathbb{R}_{+}$as in (7). The maturation takes a deterministic time $\tau$ given by (9). A particle which begins its maturation at time $T$ can either die with probability $1-p(T)$ where $p(T)$ is as in (8), or survive with probability $p(T)$ and achieve its maturation at time $T+\tau$. Now, consider independent particles which begin their maturation at times $T_{0}, T_{1}, \ldots$ These particles survive with probabilities $p\left(T_{0}\right), p\left(T_{1}\right), \ldots$, and achieve their maturation at times $M_{0}=$ $T_{0}+\tau, M_{1}=T_{1}+\tau, \ldots$ provided that the corresponding $p\left(T_{i}\right)$ are non null. The survival durations after maturation of the mature particles are i.i.d. random variables with common rate $\mu: \mathbb{R}_{+} \rightarrow \mathbb{R}_{+}$ where $\mu(t):=\kappa(t, 1)$. Suppose that the initial times $T_{0}, T_{1}, \ldots$ are random and distributed according to an independent Poisson point process on $\mathbb{R}$ with intensity $\lambda: \mathbb{R} \rightarrow \mathbb{R}_{+}$. For any $t \geqslant 0$, let $N_{t}$ be the number of mature particles still alive at time $t$. Theorem 2.2, together with (8) and (9), yields the following result.

Theorem 2.4. For any $0 \leqslant s \leqslant t$ and any $m \in \mathbb{N}, \mathcal{L}\left(N_{t} \mid N_{s}=m\right)=\mathcal{B}(m, \alpha(s, t)) * \mathcal{P}(\beta(s, t))$, where

$$
\alpha(s, t):=\exp \left(-\int_{s}^{t} \mu(w) d w\right)
$$

and

$$
\beta(s, t):=\int_{s-\tau}^{t-\tau} \lambda(u) \exp \left(-\int_{0}^{\tau} g(u+w, \rho w) d w\right) \alpha(u+\tau, t) d u .
$$

In particular, for any $0 \leqslant s \leqslant t$ and any $m \in \mathbb{N}$,

$$
\mathbf{E}\left(N_{t} \mid N_{s}=m\right)=m \alpha(s, t)+\beta(s, t) .
$$

The process $\left(N_{t}\right)_{t \geqslant 0}$ is a time inhomogeneous $\mathrm{M} / \mathrm{M} / \infty$ queue, which is a particular birth and death process, see 40, 2, 44. In an $\mathrm{M} / \mathrm{M} / \infty$ queue, each client is immediately served by an independent dedicated server. When $g \equiv 0$ and $\mu$ is constant, one has $p \equiv 1$, and $\left(N_{t}\right)_{t \geqslant 0}$ is in that case an $\mathrm{M} / \mathrm{M} / \infty$ queue with input intensity $\lambda$ and constant output intensity $\mu$. When in addition $\lambda$ is constant, 
the symmetric invariant measure of this queue is the Poisson law $\mathcal{P}(\lambda / \mu)$. In a way, the $\mathrm{M} / \mathrm{M} / \infty$ queue with constant intensities is for the Poisson process what the Ornstein-Uhlenbeck process is for Brownian motion, see [40, Theorem 6.14].

The role played by the $\mathrm{M} / \mathrm{M} / \infty$ queueing processes in our model is due to the independence of the particles in the definition of $\left(N_{t}\right)_{t \geqslant 0}$. One can alternatively consider a non independent killing after maturation, which can lead for example to an $\mathrm{M} / \mathrm{M} / 1$ type queueing process. Unfortunately, the law at fixed time of such a process, even for the time-homogeneous case, is far more complex than the simple formula obtained in the $\mathrm{M} / \mathrm{M} / \infty$ case, see [42] and [4].

Remark 2.5 (Negative values of $\kappa$ and input rate amplification heuristics). The expression of $\beta$ above still makes sense even when $\kappa$ takes negative values on $[0,1)$. In that case, the quantity $p(u)=$ $\exp \left(-\int_{0}^{\tau} g(u+w, \rho w) d w\right)$ may exceed 1, and thus cannot be interpreted as a "survival probability". Such negative values can be seen in a way as a sort of "amplification" of the input rate instead of a killing, and can be incorporated into $\lambda$. Beware that it does not correspond to an immigration of particles in the counting process. It appears as a distortion of the input rate of the counting process.

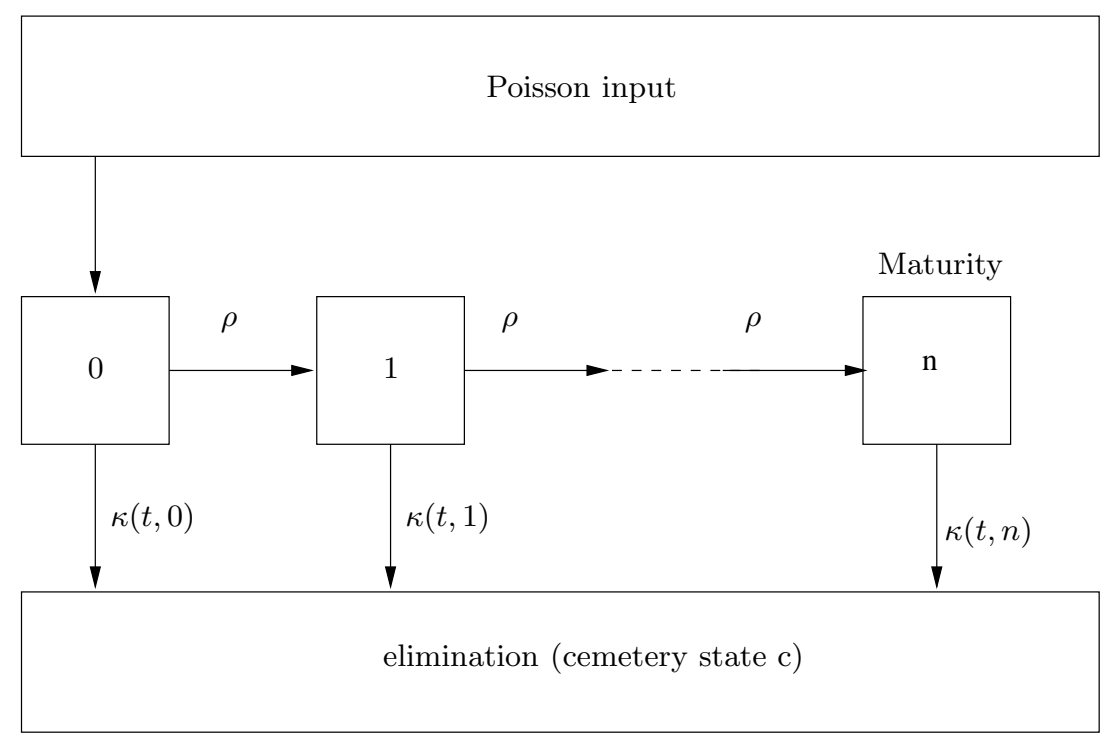

Figure 6: Catenary chain of compartments with Poisson input, one-way flow $(\rho)$, and space-time-

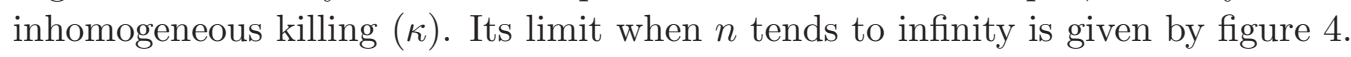

\subsubsection{Case without killing after maturation}

Assume that $\mu \equiv 0$ (no killing after maturation). In that case, $\alpha(s, t)=0$ for any $0 \leqslant s \leqslant t$, and the formula for $\beta(s, t)$ boils down to

$$
\beta(s, t)=\int_{s-\tau}^{t-\tau} \lambda(u) \exp \left(-\int_{0}^{\tau} g(u+w, \rho w) d w\right) d u .
$$

In that case, $t \in \mathbb{R}_{+} \mapsto \beta(s, t)$ is non decreasing since the main integrand does not depend on $t$. This is not surprising since the particles are never killed after maturation. Hence, on $\left\{N_{s}=0\right\}$, the process $t \in \mathbb{R}_{+} \mapsto N_{t}$ is non decreasing, and in particular its expected value $\beta(s, t)$ is non decreasing too. When $\lambda$ is constant and $g \equiv 0$, we recover a simple Poisson process of intensity $\lambda$. 


\subsubsection{Case without killing during maturation}

Assume that $g \equiv 0$ (no killing during maturation). In that case, the formula for $\beta(s, t)$ for $0 \leqslant s \leqslant t$ boils down to

$$
\beta(s, t)=\int_{s-\tau}^{t-\tau} \lambda(u) \alpha(u+\tau, t) d u .
$$

When in addition both $\lambda$ and $\mu$ are constant, we recover the standard time-homogeneous M/M/ $\infty$ queue with input rate $\lambda$ and service rate $\mu$, for which

$$
\alpha(s, t)=e^{-(t-s) \mu} \quad \text { and } \quad \beta(s, t)=\left(1-e^{-(t-s) \mu}\right) \frac{\lambda}{\mu} .
$$

The Poisson measure $\mathcal{P}(\lambda / \mu)$ is a stationary distribution of $\left(N_{t}\right)_{t \geqslant 0}$ in that case.

\subsubsection{Partial killing during maturation and constant killing after maturation}

Let us consider now the particular case where $\lambda$ is constant, and $\kappa$ is of the form

$$
\kappa(t, x)=g(t) \mathrm{I}_{[0, \delta)}(x)+\mu \mathrm{I}_{\{1\}}(x),
$$

where $\delta \in[0,1]$, where $\mu \in \mathbb{R}_{+}$, and where $g: \mathbb{R}_{+} \rightarrow \mathbb{R}_{+}$is a smooth function. It corresponds to a time dependent killing before the maturation stage $\delta$, and to a constant killing after maturation. No killing is made between maturation stage $\delta$ and full maturation. The formula for $\beta(s, t)$ when $0 \leqslant s \leqslant t$ boils down to

$$
\beta(s, t)=\lambda \int_{s-\tau}^{t-\tau} \exp \left(-\mu(t-\tau-u)-\int_{0}^{\delta \tau} g(u+w) d w\right) d u
$$

When $g \equiv 0$, this formula reduces to the classical $\mathrm{M} / \mathrm{M} / \infty$ average queue length (11). Assume instead that function $g$ in (12) only vanishes at infinity. Then the two formulas (13) and (11) for $\beta$ are equivalent when $t$ goes to $+\infty$. In particular, the Poisson measure $\mathcal{P}(\lambda / \mu)$ is a stationary distribution of $\left(N_{t}\right)_{t \geqslant 0}$ in that case. One can see on figure 0 that this Poisson equilibrium is quickly reached. It is possible to quantify the speed of convergence in total variation norm or in entropy, see [4, 40].

\subsubsection{Identifiability and invariance by some transformations}

The dynamics of $N:=\left(N_{t}\right)_{t \geqslant 0}$ is fully described by the quadruple $(\tau, \mu, g, \lambda)$, where $\tau:=1 / \rho$. It is quite natural to ask about the injectivity of the map

$$
(\tau, \mu, g, \lambda) \mapsto \mathcal{L}\left(N \mid N_{0}\right) .
$$

According to Theorem 2.4, the law $\mathcal{L}\left(N \mid N_{0}\right)$ is completely prescribed by the triple $\left(\tau, \mu, \lambda_{g}\right)$, where $\lambda_{g}: \mathbb{R} \rightarrow \mathbb{R}_{+}$is defined by

$$
\lambda_{g}(t):=\lambda(t) p(t)=\lambda(t) \exp \left(-\int_{0}^{\tau} g(t+w, \rho w) d w\right) .
$$

Thus, the couple $(\lambda, g)$ cannot be identified, since it acts on the dynamics via the compound $\lambda_{g}$. The action of $g$ can be compensated by $\lambda$ and vice versa. Namely, suppose that $g$ can be written $g=g_{1}+g_{2}$, where $g_{1}$ and $g_{2}$ are non-negative functions. Then, the two models corresponding respectively to $(\tau, \mu, g, \lambda)$ and $\left(\tau, \mu, g_{2}, \lambda_{g_{1}}\right)$ are indistinguishable. The extreme case corresponds to $\left(\tau, \mu, 0, \lambda_{g}\right)$, for which the entire killing function $g$ is merged into the input rate function $\lambda$. Let us analyze some special cases. For any $\theta>1$, let us consider the transformation which replaces $(\lambda, g)$ by $\left(\lambda^{\theta}, g^{\theta}\right)$ defined by

$$
\lambda^{\theta}:=\theta \lambda \quad \text { and } \quad g^{\theta}:=g+\rho \log (\theta) .
$$

Function $\mu$ and parameter $\rho$ are left unchanged, and one can check that $\lambda_{g^{\theta}}^{\theta}=\lambda_{g}$. Therefore, the dynamics is invariant by this transformation: the models corresponding to $(\tau, \mu, g, \lambda)$ and $\left(\tau, \mu, g^{\theta}, \lambda^{\theta}\right)$ 


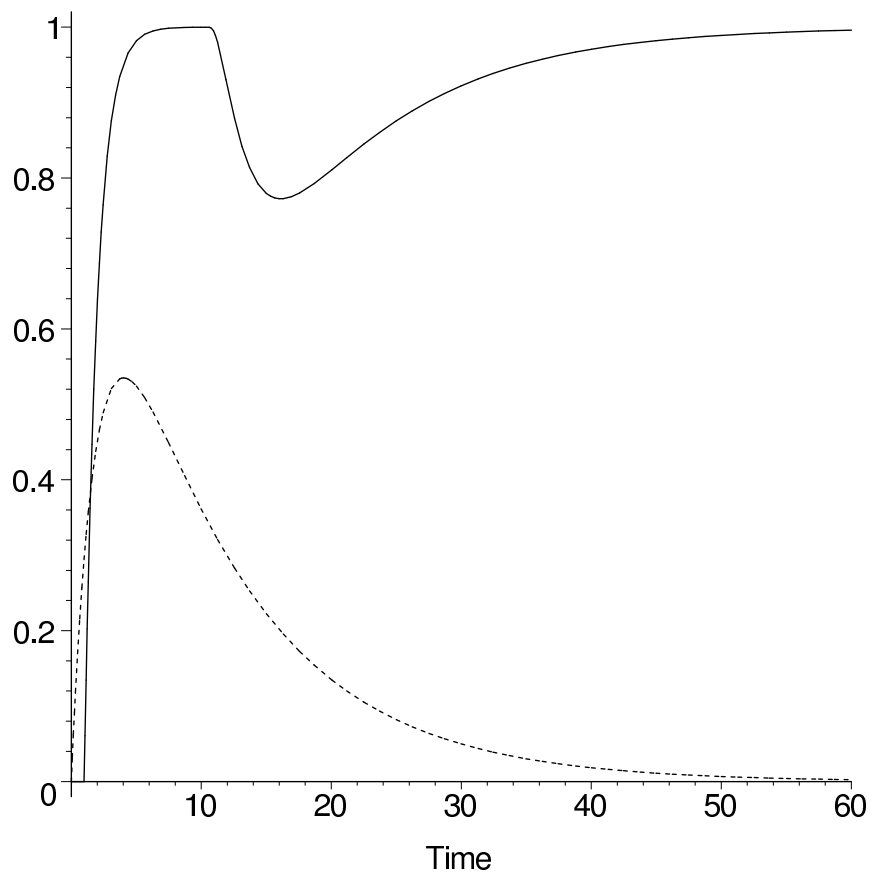

Average number of particles $\mathrm{E}(\mathrm{N}(\mathrm{t}) \mid \mathrm{N}(0)=0)$

Figure 7: The plots represent the average number $\mathbb{E}\left(N_{t} \mid N_{0}=0\right)$ of Theorem 2.4, and the killing function $g$. This example corresponds to the case (12) with $\lambda(t)=\mathrm{I}_{\mathbb{R}_{+}}(t), \alpha=\frac{1}{2}, \mu \equiv 1, \rho=1$, and $g(t-10)=\left(e^{-t / 10}-e^{-t / 2}\right) \mathrm{I}_{\mathbb{R}_{+}}(t)$. One can observe on the plot of the average number of particles three main time phases. The first phase corresponds to an ascendancy to a Poisson equilibrium before the action of the drug via $g$. The second phase corresponds to a decrease due to the action of the killing via function $g$ (delayed by the time lag is $\tau=\rho^{-1}=1$ ). In the third phase, the killing action decreases and the Poisson equilibrium is reached again.

are indistinguishable, despite their distinct physiological meanings. A multiplicative perturbation of the input intensity $\lambda$ corresponds to an additive perturbation of the killing function $g$ during maturation. Hence, one can decide to normalize the parametrization by taking for example $\lambda \equiv 1$. Namely, $(\tau, \mu, g, \lambda)$ is indistinguishable from $(\tau, \mu, G, 1)$ where

$$
G(v, y)=g(v, y)-\frac{1}{\tau} \log \lambda\left(v-\frac{y}{\rho}\right) .
$$

\section{A pharmacokinetics/pharmacodynamics example in cancerology}

Catenary chains of compartments, as depicted in figure 5, are widely used in the literature by kineticists for the modelling of anticancer drug toxicity, see for instance [6, 17, 14, 15, 16, 17] and references therein. Most anticancer drugs have toxic effects on white blood cells (myelosuppression). Neutrophils are particular white blood cells used as toxicity markers. The catenary chain of compartments models the maturation process of neutrophils in the bone marrow. Each maturation stage corresponds to a specific position in the chain. The killing rate during maturation corresponds to the drug toxicity on neutrophils. The last compartment of the chain is the only observed (blood), and the others correspond to hidden positions (bone marrow). In practice, the input and transit rates in the catenary chain of compartments are unknown parameters. The goal of the kineticist is thus to control the content of the last compartment by his action on the anticancer drug dosage regimen. Consider for simplicity that both the production rate $\lambda$, the transit rate $\rho$, and the elimination rate in blood $\mu$ are constant. The 
killing rate $\kappa$ depends typically on the time profile $q: \mathbb{R}_{+} \rightarrow \mathbb{R}$ of the anticancer drug concentration in blood (i.e. kinetics). The first step of the Pharmacokinetics/Pharmacodynamics study is to find, for a given kinetics, the best values of the parameters in view of the data (in blood). This first step can be addressed in several ways.

Approach (I). This traditional approach involves a deterministic finite catenary chain of $n$ compartments, as presented in example 1.1 page 3. It is customary to model the toxic effect of the anticancer drug on neutrophils via a killing rate $\kappa(t, i)=\gamma q(t)$ for $i \leqslant n_{0}<n, \kappa(t, n)=\mu$, and 0 elsewhere. The vector $Q(t):=\left(Q_{1}(t), \ldots, Q_{n}(t)\right)$, which represents the number of neutrophils in each compartments of the chain, solves a system of $n$ ordinary differential equations (ODE). The available data concerns only the last compartment (blood), labelled $n$. The function $t \mapsto Q_{n}(t)$ depends on the modelling parameters $\lambda, \rho, \mu, \gamma, n_{0}$. The main drawback of this approach is that the number of compartments $n$ is unknown, and that the system of equation is heavy.

Approach (II). In this approach, we circumvent the problem of the choice of $n$ by considering the continuous limit in $n$, as presented in Section 1.2. This limit procedure leads to killing rates $\kappa$ as in equation (团). More precisely, we mimic the cutoff used in the approach (I) by considering equation (12) with $g(t, x)=\gamma q(t) \mathrm{I}_{[0, \delta]}(x)$ where $\gamma>0$ and where $\delta \in[0,1)$ are constants. The continuous limit in $n$ is constructed at the level of the stochastic interpretation. As a consequence, the relevant quantity is the mean of the counting process of Theorem 2.4, given by equation (10). The initial value $m$ in (10) must be averaged with respect to the equilibrium without drug, i.e. the Poisson measure $\mathcal{P}(\lambda / \mu)$. The quantity $Q_{n}(t)$ of the approach (I) is thus replaced now by the following explicit alternative quantity:

$$
Q_{\infty}(t):=\alpha(0, t)+\beta(0, t) \frac{\lambda}{\mu},
$$

where the functions $\alpha$ and $\beta$ are as in Theorem 2.4.

Remark 3.1. Actually, one can use a Hill-type transform (see for instance [30]) to model the dependency of the killing rate with respect to the drug kinetics. However, for low drug concentration values, it is customary to simplify the approach by considering a linear dependency with a possible cutoff in space, as in the approaches (I) and (II) above. We emphasize the fact that even if the instantaneous killing rate depends linearly on the drug kinetics, the global killing effect is nonlinear with respect to the kinetics.

We compare below, on a simple example, the two approaches defined above. The approach (I) leads to the resolution of a system of ODE, whereas the approach (II) leads to the computation of explicit integral transforms given by (14).

In order to mimic practical situations, we used a synthetic dataset obtained by perturbing a dataset extracted from [6] (we did not have the permission to use directly the dataset of [6]). In this study, an anticancer drug was administrated by 30-min intravenous infusions to patients, on five consecutive days, see figure $\mathrm{B}$. For a typical patient, the drug concentration at time $t$ was proportional to :

$$
q(t)=\sum_{d=0}^{4}\left(1-e^{-\alpha(t-24 d)}\right) \mathrm{I}_{24 d+[0,1 / 2]}(t)+\left(1-e^{-\alpha / 2}\right) e^{-\beta(t-24 d-1 / 2)} \mathrm{I}_{24 d+[1 / 2, \infty]}(t),
$$

with $\alpha=1.86$ and $\beta=0.51$. For simplicity, we restrict our analysis in the sequel to a single patient. We first analyzed this dataset using approach (I) and approach (II), as described above. Let us denote by $Y_{j}$ the observed neutrophils counts in blood at time $t_{j}$.

Approach (I). The vector valued function $t \mapsto\left(Q_{1}(t), \ldots, Q_{n}(t)\right)$ solves the following system of 
ordinary differential equations:

$$
\left\{\begin{aligned}
\partial_{t} Q_{1}(t) & =\lambda-\rho Q_{1}(t)-\gamma q(t) Q_{1}(t) \\
\partial_{t} Q_{2}(t) & =\rho Q_{1}(t)-\rho Q_{2}(t)-\gamma q(t) Q_{2}(t) \\
& \vdots \\
\partial_{t} Q_{n_{0}}(t) & =\rho Q_{n_{0}-1}(t)-\rho Q_{n_{0}}(t)-\gamma q(t) Q_{n_{0}}(t) \\
& \vdots \\
\partial_{t} Q_{n}(t) & =\rho Q_{n-1}(t)-\mu Q_{n}(t)
\end{aligned}\right.
$$

At time $t=0$, the drug concentration $q$ is null, and the system is at equilibrium. Consequently, $\partial_{t=0} Q_{i}=0$ for $i \in\{1, \ldots, n\}$. The initial condition is thus $Q_{1}(0)=\cdots=Q_{n-1}(0)=\lambda / \rho$, and $Q_{n}(0)=\lambda / \mu$. These systems were numerically integrated using the ForTRAN package ODEPACK, see for instance [39, 38, 22]. Models with $\mathrm{n}=5,10,30$ and 100 compartments were used. For each $n$-compartments model, the parameters $\left(\lambda, \rho, \mu, \gamma, n_{0}\right)$ were estimated with ordinary least-squares :

$$
\arg \inf _{\lambda, \rho, \mu, \gamma, n_{0}} \sum_{j}\left(Y_{j}-Q_{n}\left(t_{j}\right)\right)^{2} .
$$

Since $n_{0}$ is an integer, all possible values of $n_{0}$, from 1 to $n-1$, were screened and the value of $n_{0}$ giving the minimum residual sum of squares was then selected.

Approach (II). The explicit expression of (14) provides the following formula for the average number of neutrophils in blood at time $t$ :

$$
Q_{\infty}(t)=\frac{\lambda}{\mu} e^{-\mu t}+\lambda e^{\mu(t-\tau)} \int_{-\tau}^{t-\tau} \exp \left(\mu u-\gamma \int_{0}^{\delta \tau} q(u+w) d w\right) d u,
$$

where $\tau=1 / \rho$. Since $q$ has an easily computable primitive, the inner integral in the expression above is explicit. The outer integral was numerically evaluated using the Clenshaw-Curtis quadrature method. The parameters $(\lambda, \rho, \mu, \gamma, \delta)$ were estimated with ordinary least-squares. Note that the continuous parameter $\delta \in[0,1)$ plays here the role of the discrete parameter $n_{0}<n$ of the approach (I).

The curves obtained with the different models are represented in figure 8. On the whole, the different models approximately give the same curves. Despite its appearance, $t \mapsto Q_{\infty}(t)$ is smooth. The curves provided by the approach (I) with 100 compartments in one hand and the approach (II) in the other hand are nearly the same. The obtained residuals sum of squares are given in table 1 . The curves are given by figure \$. The best fit was obtained with the 10-compartments model. Models with more compartments, including the $\infty$ compartments model of approach (II) give approximately the same quality of fit. The number of parameter to estimate is the same for both approach (I) and (II). However, the estimation of $n_{0}$ in approach (I) leads to a large amount of extra ODE integration. The amounts of time needed for the ODE integration are at least twice as long for models with more than 10 compartments than for the integral evaluation of approach (II). Keeping in mind that such studies usually include several tens of patients, the approach (II) that we proposed offers a reasonable alternative to compartmental models.

\begin{tabular}{llllll}
\hline & $5 \mathrm{CP}$ & $10 \mathrm{CP}$ & $30 \mathrm{CP}$ & $100 \mathrm{CP}$ & Continuous \\
\hline Computation time & 0.39 & 0.99 & 11.803 & 235.12 & 0.51 \\
Sum of Squares & 0.99 & 0.25 & 0.32 & 0.32 & 0.33 \\
\hline
\end{tabular}

Table 1: The duration of the ODE/integral evaluation. For compartmental models, these durations have been obtained as $(n-1) \times$ duration for a single evaluation of the ODE to take into account the screening needed to estimate $n_{0}$. The obtained sum-of-squares are given in the second row. 


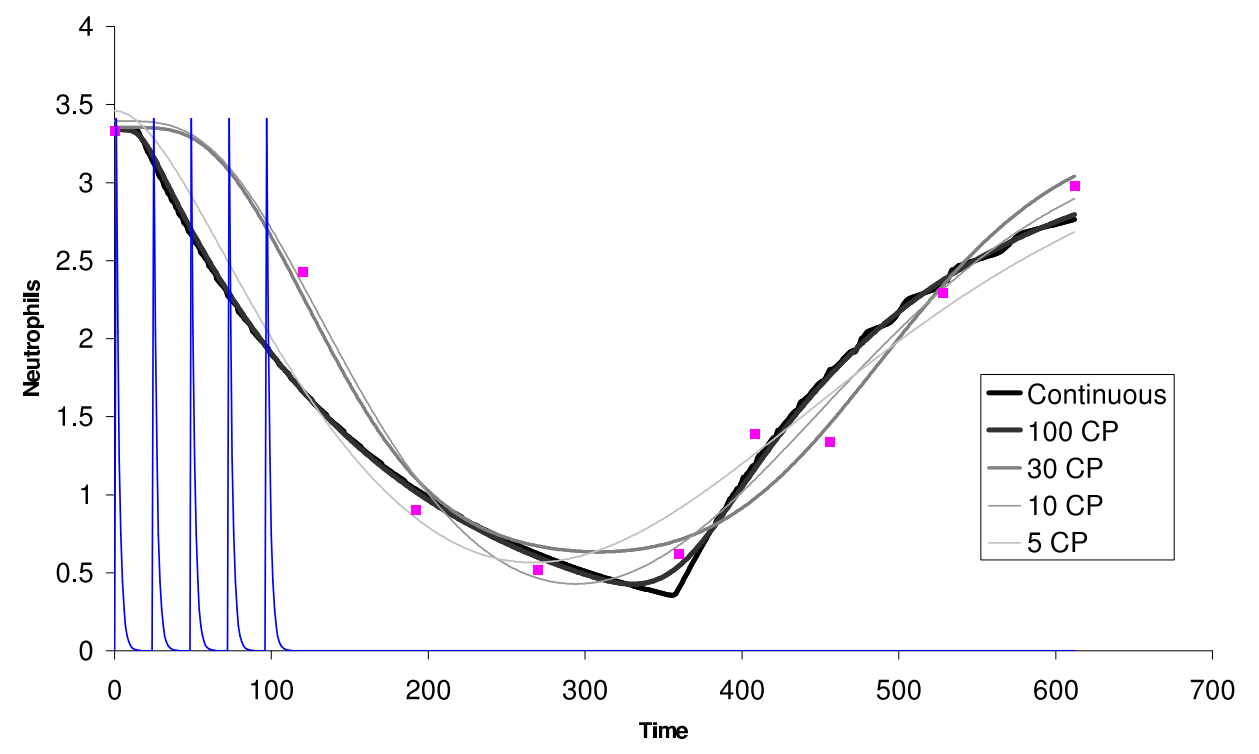

Figure 8: The curve with five peaks is the drug concentration curve $t \mapsto q(t)$ (kinetics). The neutrophils counts observed in a patient are represented by squares. The mean neutrophils counts predicted with models with 5, 10, 30, 100, $\infty$ compartments are represented with different lines. On the whole, models with at least ten compartments give a reasonable description of observed points. We emphasize that in general, the adequate number of compartments is not known a priori, and can vary from one patient to other. The estimation of an adequate number of compartments from the data has a cost. The continuous model avoids this problem.

\section{Acknowledgements}

The authors sincerely thank Prof. Laurent Miclo for stimulating and helpful discussions on inhomogeneous jump processes and related topics at the early development of this work. The article greatly benefited from the comments of two anonymous reviewers.

\section{References}

[1] W. J. Anderson, Continuous-time Markov chains, Springer Series in Statistics: Probability and its Applications, Springer-Verlag, New York, 1991, An applications-oriented approach.

[2] K. Borovkov, Elements of stochastic modelling, World Scientific Publishing Co. Inc., River Edge, NJ, 2003.

[3] P. Caputo and G. Posta, Entropy dissipation estimates in a zero-range dynamics, Probab. Theory Related Fields 139 (2007), no. 1-2, 65-87.

[4] D. Chafaï, Binomial-Poisson entropic inequalities and the $M / M / \infty$ queue, ESAIM Probab. Stat. 10 (2006), 317-339 (electronic).

[5] H. Chen and D. D. Yao, Fundamentals of queueing networks, Applications of Mathematics (New York), vol. 46, Springer-Verlag, New York, 2001, Performance, asymptotics, and optimization, Stochastic Modelling and Applied Probability.

[6] D. Concordet, F. Léger, and C. Ané, Population PK/PD Analysis, Encyclopedia of Biopharmaceutical Statistics, Dekker, 2004.

[7] N.L. Dayneka, V. Garg, and W.J. Jusko, Comparison of four basic models of indirect pharmacodynamic responses, J. Pharmacokin. Biopharm. (1993), no. 21, 457-478.

[8] P. Del Moral, Feynman-Kac formulae, Probability and its Applications (New York), Springer-Verlag, New York, 2004, Genealogical and interacting particle systems with applications.

[9] R. Durrett, Ten lectures on particle systems, Lectures on probability theory (Saint-Flour, 1993), Lecture Notes in Math., vol. 1608, Springer, Berlin, 1995, pp. 97-201. 
[10] A. Economou, The compound Poisson immigration process subject to binomial catastrophes, J. Appl. Probab. 41 (2004), no. 2, 508-523.

[11] S.N. Ethier and T.G. Kurtz, Markov processes, Wiley Series in Probability and Mathematical Statistics: Probability and Mathematical Statistics, John Wiley \& Sons Inc., New York, 1986, Characterization and convergence.

[12] G. Fayolle, V. A. Malyshev, and M. V. Men'shikov, Topics in the constructive theory of countable Markov chains, Cambridge University Press, Cambridge, 1995.

[13] R. D. Foley, The non-homogeneous $M / G / \infty$ queue, Operations Res. 19 (1982), 40-48.

[14] L.E. Friberg, C.J. Brindley, M.O. Karlsson, and A.J. Devlin, Models of schedule dependent haematological toxicity of 2'-deoxy-2'-methylidenecytidine (DMDC), Eur. J. Clin. Pharmacol. 56 (2000), no. 8, 567-74.

[15] L.E. Friberg, A. Freijs, M. Sandström, and M.O. Karlsson, Semiphysiological model for the time course of leukocytes after varying schedules of 5-fluorouracil in rats, J. Pharmacol. Exp. Therap. 2 (2000), no. 295, 734-740.

[16] L.E. Friberg and M.O. Karlsson, Mechanistic models for myelosuppression, Invest. New Drug (2003), no. 21, 183194.

[17] J.V.S. Gobburu and W.J. Jusko, Role of dosage regimen in controlling indirect pharmacodynamic responses, Advanced Drug Delivery Reviews (2001), no. 46, 45-57.

[18] J. Goudriaan, Boxcartrain methods for modelling of ageing, development, delays and dispersion, The dynamics of physiologically structured populations (Amsterdam, 1983), Lecture Notes in Biomath., vol. 68, Springer, Berlin, 1986, pp. 453-473.

[19] Jan Grandell, Mixed Poisson processes, Monographs on Statistics and Applied Probability, vol. 77, Chapman \& Hall, London, 1997.

[20] I. Győri, Interconnection between ordinary and delay differential equations, Modern optimal control, Lecture Notes in Pure and Appl. Math., vol. 119, Dekker, New York, 1989, pp. 131-141.

[21] J. Michael Harrison and Austin J. Lemoine, A note on networks of infinite-server queues, J. Appl. Probab. 18 (1981), no. 2, 561-567.

[22] A. C. Hindmarsh, ODEPACK, a systematized collection of ODE solvers, Scientific computing (Montreal, Que., 1982), IMACS Trans. Sci. Comput., I, IMACS, New Brunswick, NJ, 1983, pp. 55-64.

[23] J. R. Jackson, Networks of waiting lines, Operations Res. 5 (1957), 518-521.

[24] _ Jobshop-like Queuing Systems, Management Sciences 10 (1963), no. 1, 131-142.

[25] J.A. Jacquez, Density functions of residence times for deterministic and stochastic compartmental systems, Math. Biosci. 180 (2002), 127-139, John A. Jacquez memorial volume.

[26] J.A. Jacquez and C.P. Simon, Qualitative theory of compartmental systems, SIAM Rev. 35 (1993), no. 1, 43-79.

[27] _ Qualitative theory of compartmental systems with lags, Math. Biosci. 180 (2002), 329-362, John A. Jacquez memorial volume.

[28] I. Karatzas and S.E. Shreve, Brownian motion and stochastic calculus, second ed., Graduate Texts in Mathematics, vol. 113, Springer-Verlag, New York, 1991.

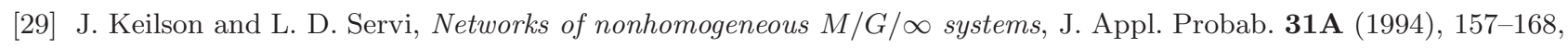
Studies in applied probability.

[30] F. P. Kelly, Reversibility and stochastic networks, John Wiley \& Sons Ltd., Chichester, 1979, Wiley Series in Probability and Mathematical Statistics.

[31] J. F. C. Kingman, Poisson processes, Oxford Studies in Probability, vol. 3, The Clarendon Press Oxford University Press, New York, 1993, , Oxford Science Publications.

[32] C. Kipnis and C. Landim, Scaling limits of interacting particle systems, Grundlehren der Mathematischen Wissenschaften [Fundamental Principles of Mathematical Sciences], vol. 320, Springer-Verlag, Berlin, 1999.

[33] T. M. Liggett, Interacting particle systems, Classics in Mathematics, Springer-Verlag, Berlin, 2005, Reprint of the 1985 original.

[34] N. Limić, Relationship between compartmental masses and transition rates in stochastic compartmental models, Math. Biosci. 114 (1993), no. 1, 59-79.

[35] J. H. Matis and T. R. Kiffe, Stochastic population models, Lecture Notes in Statistics, vol. 145, Springer-Verlag, New York, 2000, A compartmental perspective.

[36] J. D. Murray, Mathematical biology. I, third ed., Interdisciplinary Applied Mathematics, vol. 17, Springer-Verlag, New York, 2002, An introduction.

[37] S.-I. Niculescu and K. Gu (eds.), Advances in time-delay systems, Lecture Notes in Computational Science and Engineering, vol. 38, Springer-Verlag, Berlin, 2004. 
[38] L. Petzold, Automatic selection of methods for solving stiff and nonstiff systems of ordinary differential equations, SIAM J. Sci. Statist. Comput. 4 (1983), no. 1, 137-148.

[39] K. Radhakrishnan and A. C. Hindmarsh, Description and use of LSODE, the Livermore Solver for Ordinary Differential Equations, LLNL report UCRL-ID-113855, http://www.llnl.gov/CASC/nsde/pubs/u113855.pdf, december 1993.

[40] Ph. Robert, Stochastic networks and queues, french ed., Applications of Mathematics (New York), vol. 52, SpringerVerlag, Berlin, 2003, Stochastic Modelling and Applied Probability.

[41] K. Schuhmacher and H. Thieme, Some theoretical and numerical aspects of modelling dispersion in the development of ectotherms, Comput. Math. Appl. 15 (1988), no. 6-8, 565-594, Hyperbolic partial differential equations. V.

[42] A. M. K. Tarabia, A new formula for the transient behaviour of a non-empty $M / M / 1 / \infty$ queue, Appl. Math. Comput. 132 (2002), no. 1, 1-10.

[43] P. G. Taylor and R. J. Boucherie, Transient product from distributions in queueing networks, Discrete Event Dynamic Systems 3 (1993), no. 4, 375-396.

[44] F.J. Zhang and Y.Y. Chen, A nonhomogeneous Markov queuing process, J. Xinjiang Univ. Nat. Sci. (1985), no. 1, $1-11$.

Djalil Chafaï and Didier Concordet

Address: UMR 181 INRA/ENVT Physiopathologie et Toxicologie Expérimentales, École Nationale Vétérinaire de Toulouse,

23 Chemin des Capelles, F-31076, Toulouse CEDEx 3, France.

E-mail: mailto:d.chafai@envt.fr.nospam

Address: UMR 5583 CNRS/UPS Laboratoire de Statistique et Probabilités, Institut de Mathématiques de Toulouse, Université Paul Sabatier,

118 route de Narbonne, F-31062, Toulouse, CEDEX 4, France.

E-mail: mailto:chafai(AT)math.univ-toulouse.fr

Web: http://www.math.univ-toulouse/ ${ }^{\sim}$ chafai 